\title{
Effects of the Unburned-Gas Temperature and Lewis Number on the Intrinsic Instability of High-Temperature Premixed Flames*
}

\author{
Satoshi KADOWAKI**, Masafumi YAHATA** and Hideaki KOBAYASHI*** \\ ** Department of System Safety, Nagaoka University of Technology \\ Kamitomioka, Nagaoka 940-2188, Japan \\ E-mail: kadowaki@mech.nagaokaut.ac.jp \\ *** Institute of Fluid Science, Tohoku University \\ Katahira, Aoba-ku, Sendai 980-8577, Japan
}

\begin{abstract}
The effects of the unburned-gas temperature and Lewis number on the intrinsic instability of high-temperature premixed flames under the constant-enthalpy conditions were investigated by two-dimensional unsteady calculations of reactive flows. A sinusoidal disturbance with sufficiently small amplitude was superimposed on a planar flame to obtain the relation between the growth rate and wave number, i.e. the dispersion relation. As the unburned-gas temperature became higher, the growth rate increased and the unstable range widened. This was due to the increase of the burning velocity of a planar flame. In addition, the obtained numerical results were consistent with the theoretical solutions in small wave-number region. As the Lewis number became smaller ( larger ), the growth rate increased ( decreased) and the unstable range widened ( narrowed), which was due to diffusive-thermal effects. The dispersion relation yielded the linearly most unstable wave number, i.e. the critical wave number. The critical wave number increased as the unburned-gas temperature became higher. Thus, the critical wavelength shrank, and then the cell size shrank. To clarify the characteristics of cellular flames induced by intrinsic instability, a finite disturbance with the critical wavelength was superimposed. The superimposed disturbance evolved, and a cellular-shaped front formed. In all Lewis numbers, the behavior of cellular flames became milder as the unburned-gas temperature became higher, even though the growth rate increased. The normalized burning velocities of cellular flames decreased monotonously. This was because that the thermal-expansion effects became weaker owing to the decrease of the difference in temperature between the burned and unburned gases, which was generated by the conditions of constant enthalpy, i.e. constant burned-gas temperature.
\end{abstract}

Key words: High-Temperature Premixed Flame, Intrinsic Instability, Cellular Flame, Unburned-Gas Temperature, Lewis Number

\section{Introduction}

The irreversible process always occurs in combustion owing to the viscosity, heat conduction, mass diffusion and chemical reaction. The irreversibility generates the entropy and decreases the available energy. ${ }^{(1)-(2)}$ To reduce the exergy loss, the preheating of combustible mixtures due to the heat recirculation is often used. ${ }^{(3)-(4)}$ In view of the

${ }^{*}$ Received 25 June, 2011 (№. 11-0347) [DOI: 10.1299/jtst.6.376]

Copyright $\odot 2011$ by JSME 
energy saving, we have to investigate the reduction of irreversibility in combustion, i.e. the reduction of exergy loss.

The excess enthalpy combustion and high-temperature air combustion are well-recognized as the effective method for the reduction of exergy loss. The concept of the excess enthalpy combustion was firstly introduced by Weinberg, ${ }^{(5)}$ where combustible mixtures were preheated by the heat transfer from hot burned gases. It was shown in a series of investigations that the concept successfully extended the flammable range by the heat recirculation. In addition, we have several fundamental investigations on the excess enthalpy combustion. ${ }^{(6)-(8)}$ In the high-temperature air combustion, on the other hand, the mass recirculation, together with the heat recirculation, is adopted. Unique combustion phenomena were observed under the conditions that the temperature of combustible mixtures was sufficiently high and the oxygen concentration in mixtures was considerably low. ${ }^{(9)-(11)}$ Applying the high-temperature air combustion to industrial furnaces, more than $30 \%$ of energy saving was estimated, ${ }^{(9)}$ and the effectiveness in stoker-type incinerators was reported. ${ }^{(12)}$

To understand in detail the excess enthalpy combustion and high-temperature air combustion, we have to obtain the fundamental knowledge on the characteristics of flames, e.g. the burning velocity, flame instability, flame structure, front behavior, and so on. The burning velocity occupies a significant position in the characteristics of flames with the heat and/or mass recirculation, and the flame instability affects the structure of flames and controls the behavior of fronts. The burning velocity and intrinsic instability of premixed flames with high-temperature combustible mixtures, so-called high-temperature premixed flames, were analytically investigated. ${ }^{(13)}$ The unburned-gas temperature has a great influence on the burning velocity and on the intensity of instability. Therefore, we strongly desire to elucidate the mechanism of intrinsic instability of high-temperature premixed flames.

We have three basic types of intrinsic instability associated with premixed flames, i.e. hydrodynamic effects, diffusive-thermal effects, and body-force effects. ${ }^{(14)}$ In these effects, both of hydrodynamic effects and diffusive-thermal effects are most significant for the flame instability. Hydrodynamic effects are caused by the thermal expansion of combustible mixtures through flame fronts and are essential to the intrinsic instability of premixed flames. Diffusive-thermal effects are caused by the preferential diffusion of heat and mass, and have a destabilizing (stabilizing) influence when the Lewis number of the deficient reactant is smaller (larger) than unity. Thus, diffusive-thermal instability appears at $L e<1$.

The flame instability was analytically investigated by Sivashinsky ${ }^{(15)}$ and Joulin-Clavin, ${ }^{(16)}$ based on the diffusive-thermal model equation disregarding thermal expansion. After that, linear analyses on the instability of premixed flames considering thermal expansion were performed by Pelce-Clavin ${ }^{(17)}$ and Frankel-Sivashinsky. ${ }^{(18)}$ The effects of thermal expansion and Lewis number on instability were estimated. The previous analytical results are adequate for sufficiently small disturbances, i.e. linear region; the adopted approach is inadequate for cellular flames, i.e. non-linear region, however. Thus, we need to select the other methods to elucidate the mechanism of cellular flames induced by intrinsic instability.

Numerical approach is powerful to elucidate the instability mechanism. To investigate the intrinsic instability of premixed flames, numerical calculations were performed, based on the diffusive-thermal model equation ${ }^{(19-20)}$ and on the compressible Navier-Stokes equation. ${ }^{(21)-(25)}$ Through numerical calculations, the formation of cellular fronts induced by intrinsic instability and the mechanism of unstable behavior of premixed flames were clarified. However, previous numerical investigations did not treat high-temperature premixed flames. Thus, we should perform numerical calculations to investigate the 
intrinsic instability of high-temperature premixed flames.

The activation energy affects the flame instability drastically. Linear stability analyses of planar premixed flames with finite activation energy were performed, where cellular flames were not examined, and it was reported that the activation energy have much effects on the instability of $L e \neq 1$ flames. ${ }^{(26)}$ In addition, the activation-energy effects on the intrinsic instability of adiabatic and non-adiabatic premixed flames were investigated. ${ }^{(27)}$ Although the activation energy is constant, the Zeldovich number becomes smaller in high-temperature premixed flames. This is because that the unburned-gas temperature is high compared with usual premixed flames. The Zeldovich number plays a significant role in diffusive-thermal effects. Thus, the unburned-gas temperature is a significant factor to the characteristics of intrinsic instability, especially in $L e \neq 1$ flames. However, the intrinsic instability of high-temperature premixed flames has not yet investigated. Therefore, we have to treat high-temperature premixed flames to clarify the effects of the unburned-gas temperature and Lewis number on intrinsic instability. The numerical results on instability will be significant information for the industrial application of premixed flames with heat and mass recirculation.

In this paper, we performed numerical calculations of two-dimensional unsteady reactive flows, based on the compressible Navier-Stokes equation including a one-step irreversible chemical reaction. We investigated the effects of the unburned-gas temperature and Lewis number on the intrinsic instability of high-temperature premixed flames and clarified the characteristics of cellular flames induced by intrinsic instability.

\section{Nomenclature}

$a$

$a_{\mathrm{i}}$

$B$

$C_{\mathrm{p}}$

$c_{0}$

D

E

$e$

$k$

$k_{\mathrm{c}}$

Le

$M_{0}$

$m_{\mathrm{u}}$

Pr

$p$

$p_{\mathrm{u}}$

$p_{0}$

$Q$

$R$

$S_{\text {cf }}$

$S_{\mathrm{u}}$

$S_{\mathrm{u} 0}$

$T$

$T_{\mathrm{b}}$

$T_{\mathrm{u}}$ non-dimensional amplitude of a disturbance, referred to as $\delta$

non-dimensional initial amplitude of a disturbance, referred to as $\delta$

non-dimensional frequency factor of the reaction rate, referred to as $S_{\mathrm{u} 0} / \delta$

specific heat at constant pressure

sound velocity of the unburned gas for standard premixed flames $\left(=\sqrt{ } \gamma p_{0} / \rho_{0}\right)$

diffusion coefficient

non-dimensional activation energy, referred to as $R T_{0}$

non-dimensional stored energy ( $=$ inertial energy + kinetic energy $)$, referred to

as $p_{0}$

non-dimensional wave number, referred to as $1 / \delta$

non-dimensional critical wave number $\left(=2 \pi / \lambda_{\mathrm{c}}\right)$, referred to as $1 / \delta$

Lewis number $(=\alpha / D)$

Mach number of the burning velocity for standard premixed flames ( $\left.=S_{\mathrm{u} 0} / c_{0}\right)$

non-dimensional mass flux density $\left(=\rho_{\mathrm{u}} S_{\mathrm{u}}\right)$, referred to as $\rho_{0} S_{\mathrm{u} 0}$

Prandtl number $(=v / \alpha)$

non-dimensional pressure, referred to as $p_{0}$

non-dimensional pressure of the unburned gas, referred to as $p_{0}$

pressure of the unburned gas for standard premixed flames

non-dimensional heating value, referred to as $C_{\mathrm{p}} T_{0} / Y_{0}$

universal gas constant

non-dimensional burning velocity of a cellular flame, referred to as $S_{\mathrm{u} 0}$

non-dimensional burning velocity of a planar flame, referred to as $S_{\mathrm{u} 0}$

burning velocity for standard premixed flames

non-dimensional temperature, referred to as $T_{0}$

non-dimensional temperature of the burned gas, referred to as $T_{0}$

non-dimensional temperature of the unburned gas, referred to as $T_{0}$ 
$T_{0} \quad$ temperature of the unburned gas for standard premixed flames

$t \quad$ non-dimensional time, referred to as $\delta / S_{\mathrm{u} 0}$

$u, v \quad$ non-dimensional velocities in $x$ - and $y$-directions, referred to as $S_{\mathrm{u} 0}$

$x, y \quad$ coordinates

$Y \quad$ non-dimensional mass fraction of fuel, referred to as $Y_{0}$

$Y_{\mathrm{u}} \quad$ non-dimensional mass fraction of fuel in the unburned gas, referred to as $Y_{0}$

$Y_{0} \quad$ mass fraction of fuel in the unburned gas for standard premixed flames

Greek

$\alpha \quad$ thermal diffusivity

$\gamma \quad$ ratio of two specific heats

$\delta \quad$ preheat zone thickness for usual premixed flames $\left(=\alpha / S_{\mathrm{u} 0}\right)$

$\lambda \quad$ non-dimensional wavelength, referred to as $\delta$

$\lambda_{\mathrm{c}} \quad$ non-dimensional critical wavelength $\left(=2 \pi / k_{\mathrm{c}}\right)$, referred to as $\delta$

$v \quad$ kinematic viscosity

$\rho \quad$ non-dimensional density, referred to as $\rho_{0}$

$\rho_{\mathrm{u}} \quad$ non-dimensional density of the unburned gas, referred to as $\rho_{0}$

$\rho_{0} \quad$ density of the unburned gas for standard premixed flames

$\omega \quad$ non-dimensional growth rate, referred to as $S_{\mathrm{u} 0} / \delta$

\section{Governing Equations}

We dealt with single-reactant flames, where the abundant reactant was excessive and the chemical reaction was controlled only by the deficient reactant. The following assumptions were used: The chemical reaction was a one-step irreversible exothermic reaction, and the reaction rate obeyed the Arrhenius' equation and depended on the mass fraction of fuel and temperature. The unburned and burned gases had the same molecular weights and the same Lewis numbers, and the ideal gas equation of state was satisfied. The specific heat and transport coefficients, e.g. dynamic viscosity and thermal conductivity, were independent of temperature. The temperature dependence had no essential influence on intrinsic instability. The body force, Soret effect, Dufour effect, pressure gradient diffusion, bulk viscosity and heat loss were all negligible.

We used the compressible Navier-Stokes equation to take account of hydrodynamic effects which largely contribute to the intrinsic instability of all premixed flames. The viscous term in the equation of energy conservation was disregarded, because its contribution was trivial in the problem. Using Cartesian coordinates, we took the direction tangential to the flame front as the $y$-direction, with the gas velocity in the positive $x$-direction. The flow variables were non-dimensionalized by the physical quantities of usual premixed flames, i.e. the density, pressure and temperature of the unburned gas, the burning velocity, and the preheat zone thickness.

The governing equations of two-dimensional unsteady reactive flows were written in the conservation form as

$$
\frac{\partial \mathbf{U}}{\partial t}+\frac{\partial \mathbf{F}}{\partial x}+\frac{\partial \mathbf{G}}{\partial y}=\mathbf{S}
$$

where $\mathbf{U}, \mathbf{F}, \mathbf{G}$, and $\mathbf{S}$ are vectors given by 


$$
\mathbf{U}=\left(\begin{array}{c}
\rho \\
\rho u \\
\rho v \\
e \\
\rho Y
\end{array}\right)
$$

$$
\mathbf{F}=\left(\begin{array}{c}
\rho u \\
\rho u^{2}+\frac{p}{\gamma M_{0}^{2}}-\operatorname{Pr}\left(\frac{4}{3} \frac{\partial u}{\partial x}-\frac{2}{3} \frac{\partial v}{\partial y}\right) \\
\rho u v-\operatorname{Pr}\left(\frac{\partial v}{\partial x}+\frac{\partial u}{\partial y}\right) \\
(e+p) u-\frac{\gamma}{\gamma-1} \frac{\partial T}{\partial x} \\
\rho Y u-\frac{1}{L e} \frac{\partial Y}{\partial x}
\end{array}\right)
$$

$$
\mathbf{G}=\left(\begin{array}{c}
\rho v \\
\rho u v-\operatorname{Pr}\left(\frac{\partial v}{\partial x}+\frac{\partial u}{\partial y}\right) \\
\rho v^{2}+\frac{p}{\gamma M_{0}^{2}}-\operatorname{Pr}\left(\frac{4}{3} \frac{\partial v}{\partial y}-\frac{2}{3} \frac{\partial u}{\partial x}\right) \\
(e+p) v-\frac{\gamma}{\gamma-1} \frac{\partial T}{\partial y} \\
\rho Y v-\frac{1}{L e} \frac{\partial Y}{\partial y}
\end{array}\right)
$$

$$
\mathbf{S}=\left(\begin{array}{c}
0 \\
0 \\
0 \\
\frac{\gamma}{\gamma-1} Q B \rho Y \exp (-E / T) \\
-B \rho Y \exp (-E / T)
\end{array}\right)
$$

The ideal gas equation of state is

$$
p=\rho T
$$

\section{Numerical Procedure}

We simulated a usual premixed flame whose burning velocity and adiabatic flame 
temperature were $3.93 \mathrm{~m} / \mathrm{s}\left(M_{0}=0.01\right)$ and $2086 \mathrm{~K}\left(T_{\mathrm{b}}=7.0\right)$, respectively, at room temperature and atmospheric pressure. Although the burning velocity adopted in the present calculation is large, this value is possible for hydrogen-oxygen premixed flames and is sufficiently small compared with the sound velocity. In the present problem, thus, we obtain almost the same results on instability even though the burning velocity changes.

The used parameters were $\operatorname{Pr}=1.0, \gamma=1.4, Q=6.0$ and $E=70$. The frequency factor of the reaction rate was determined under the conditions that the calculated burning velocity of a planar flame at $T_{\mathrm{u}}=1$ should be equal to unity. We took $L e=0.5,1.0$ and 1.5 to investigate diffusive-thermal effects on intrinsic instability. When $L e<1$ ( Le $>1$ ), diffusive-thermal effects have a destabilizing ( stabilizing ) influence on the flame front.

To investigate the effects of the unburned-gas temperature, we took $T_{\mathrm{u}}=1$ through 3 . The constant-enthalpy conditions were used, so that the temperature of the burned gas was constant. The following relation was satisfied:

$$
T_{\mathrm{u}}+Q Y_{\mathrm{u}}=T_{\mathrm{b}}=\text { const. }
$$

In calculations, we considered the conditions that the pressure of the unburned gas was constant $\left(p_{\mathrm{u}}=1.0\right)$.

The two-dimensional governing equations were solved by the explicit MacCormack scheme. This scheme has second-order accuracy in both time and space. The calculation domain was 200 times the preheat zone thickness in the $x$-direction and one wavelength of a disturbance in the $y$-direction. A uniformly spaced grid was used, and the grid sizes in $x$ and $y$-directions were 0.2 and $\lambda / 64$, respectively. The calculation domain was resolved by a $1001 \times 65$ grid. The used grid was fine enough to perform calculations on this type of problem. When the size of grid was halved, no significant difference was observed. A time-step interval was adopted to satisfy the Courant-Friedrichs-Lewy ( CFL ) condition, and the non-dimensional calculation time was $t=100$.

The boundary conditions in the $x$-direction were provided by free-flow conditions. The boundary conditions in the $y$-direction were provided by periodic conditions.

A disturbance periodic in the $y$-direction was superimposed on a stationary planar flame, and the evolution of disturbed flame fronts was calculated. We adopted a sinusoidal disturbance, and the displacement of the flame front in the $x$-direction due to the superimposed disturbance was

$$
a_{\mathrm{i}} \sin (2 \pi y / \lambda)
$$

The initial amplitude was set to 0.1 for calculations of the dispersion relation and to 1.0 for cellular flames.

Numerical calculations were performed by a scalar parallel computer SGI Altix3700Bx2 at Institute of Fluid Science, Tohoku University. Each computation time ranged 30 minutes to one hour.

\section{Stationary Planar Flames}

We performed numerical calculations of reactive flows to investigate the effects of the unburned-gas temperature on the characteristics of stationary planar flames. Since the constant-enthalpy conditions were used, the mass fraction of fuel decreased as the unburned-gas temperature became higher ( see Eq. 3 ). 
Figure 1 shows the distributions of flow variables in $L e=1.0$ stationary planar flames at $T_{\mathrm{u}}=1.0,2.0$ and 3.0, where $Y_{\mathrm{u}}=1.0,0.83$ and 0.67 , respectively. Because of the constant-enthalpy conditions, the burned-gas temperature is constant. Thus, the effects of thermal expansion through the flame front decrease as the unburned-gas temperature becomes higher. The Mach number is sufficiently small, and the unburned-gas pressure is set equal to 1.0. Thus, the pressure is almost unity through the whole calculation domain, and then the burned-gas density is almost constant.

Figure 2 shows the burning velocities and mass flux densities in $L e=1.0$ stationary planar flames, depending on the unburned-gas temperature. As the unburned-gas temperature becomes higher, the mass flux density increases slightly, which is due to the dependence of reaction rate on temperature. Thus, the flame thickness becomes slightly narrower ( see Fig. 1 ). The burning velocity increases drastically with an increase of the unburned-gas temperature. This is because of the increase in the mass flux density and of the decrease in the unburned-gas density under the constant-pressure conditions.
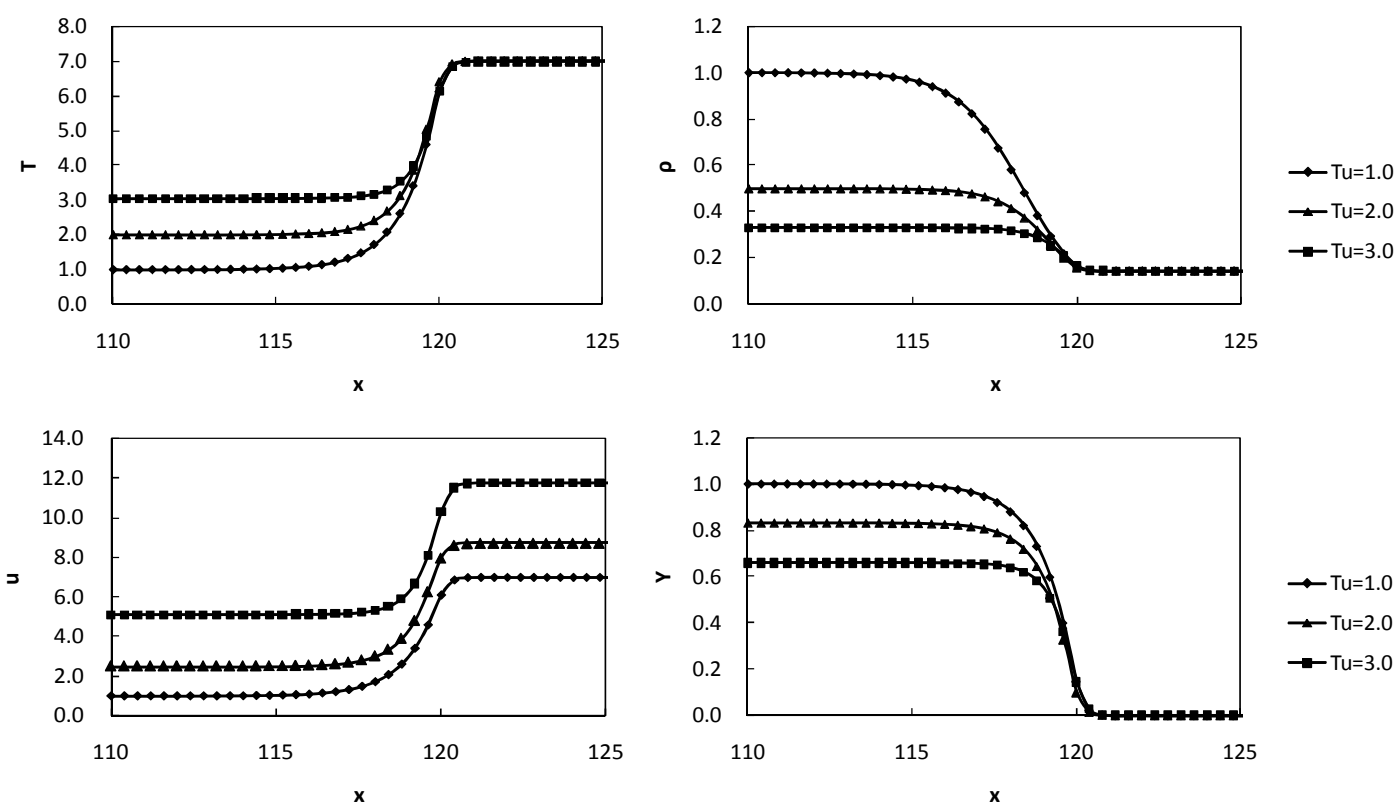

Fig. 1. Distributions of flow variables in $L e=1.0$ stationary planar flames at $T_{\mathrm{u}}=1.0,2.0$ and 3.0, where $Y_{\mathrm{u}}=1.0,0.83$ and 0.67 , respectively.

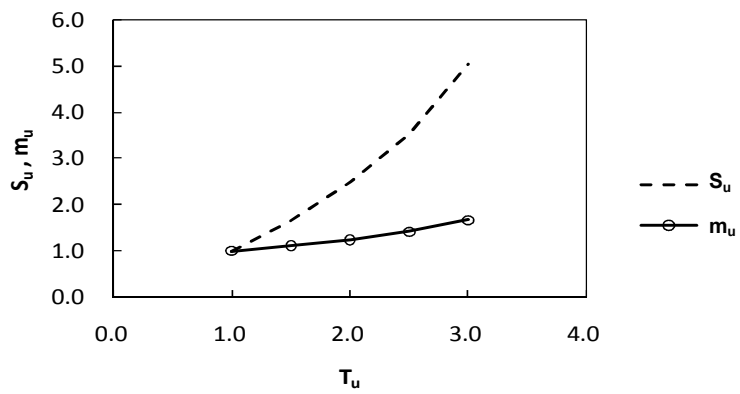

Fig. 2. Burning velocities and mass flux densities in $L e=1.0$ stationary planar flames, depending on the unburned-gas temperature. 


\section{Dispersion Relations}

To investigate the intrinsic instability of high-temperature premixed flames, a sufficiently small sinusoidal disturbance on a planar flame, where $a_{\mathrm{i}}=0.1$. The amplitude of a disturbance grows exponentially with time:

$$
a \propto \exp (\omega t)
$$

This behavior is consistent with the prediction of previous linear analyses. ${ }^{17-18}$ The behavior of the disturbed flame front, i.e. growing exponentially with time, is observed only when the amplitude is sufficiently small. When the superimposed disturbance grows to some degree, the growth rate is gradually lowered and eventually drops to zero, which is due to the nonlinearity brought about by finite amplitude.

The growth rate of a disturbance depends on the wavelength, i.e. on the wave number $(k=2 \pi / \lambda)$. The relation between the growth rate and the wave number is called the dispersion relation. Figure 3 shows the dispersion relations at $L e=1.0$ and $T_{\mathrm{u}}=1.0,2.0$ and 3.0. In this case, only hydrodynamic effects have an influence on intrinsic instability. As the unburned-gas temperature becomes higher, the growth rate increases and the unstable range widens. This is due to the increase of the burning velocity ( see Fig. 2 ). The straight lines denote the results of theoretical analyses on the dispersion relation. ${ }^{13}$ Theoretical analyses treat sufficiently thin flames, and the obtained results are valid only for sufficiently small wave numbers. In small wave-number range, the present numerical results are consistent with the theoretical solutions.

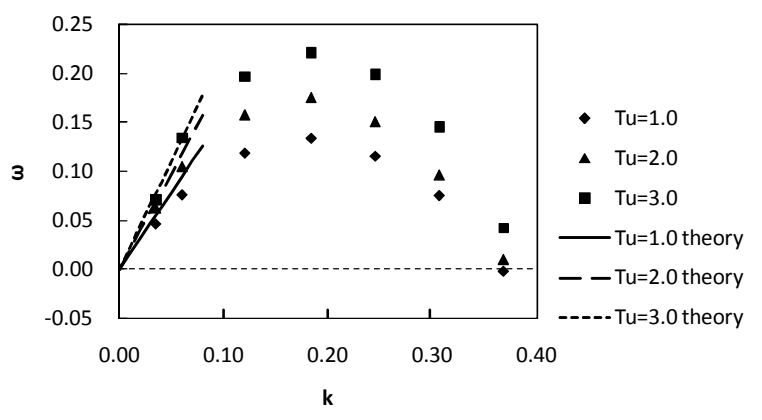

Fig. 3. Dispersion relations at $L e=1.0$ and $T_{\mathrm{u}}=1.0,2.0$ and 3.0; the straight lines denote the results of theoretical analyses on the dispersion relation.
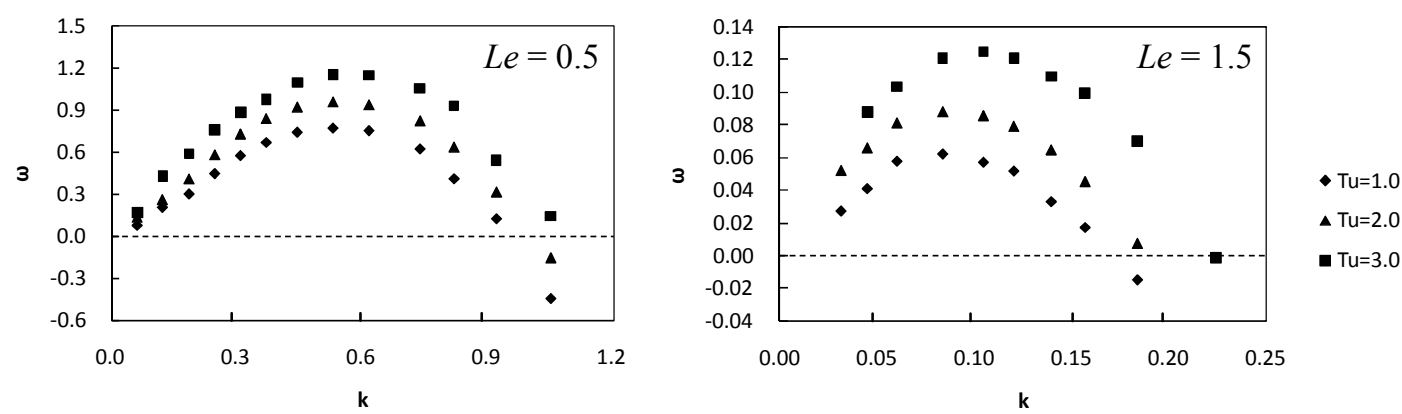

Fig. 4. Dispersion relations at $L e=0.5 \& 1.5$ and $T_{\mathrm{u}}=1.0,2.0$ and 3.0. 
The dispersion relations at $L e=0.5 \& 1.5$ are shown in Fig. 4, where diffusive-thermal effects have a destabilizing ( stabilizing ) influence on the flame instability at low ( high ) Lewis numbers. In both cases, the growth rate increases and the unstable range widens as the unburned-gas temperature becomes higher. The dispersion relation yields the wave number corresponding to the maximum growth rate, i.e. the linearly most unstable wave number, and we refer to as the critical wave number. The higher the unburned-gas temperature, the larger the critical wave number. Thus, the critical wavelength shrinks, and then the cell size shrinks.

\section{Cellular Flames}

To investigate the characteristics of cellular flames induced by intrinsic instability, we superimposed a finite disturbance $\left(a_{\mathrm{i}}=1.0\right)$ with the critical wavelength on a planar flame. Although smaller disturbances were superimposed, we obtained the same results on the formation of a cellular-shaped front.

The time histories of amplitude at $L e=1.0$ and $T_{\mathrm{u}}=1.0,2.0$ and 3.0 are shown in Fig. 5. When the amplitude is sufficiently small, it grows exponentially with time owing to hydrodynamic effects. Growing to some degree, the growth rate is gradually lowered and eventually drops to zero, and then the amplitude is almost constant. At the beginning of calculations, the growth rate of the $T_{\mathrm{u}}=3.0$ flame is greater owing to larger burning velocity, so that the amplitude increases more quickly. At the region of zero growth rates, on the other hand, the amplitude of the $T_{\mathrm{u}}=3.0$ flame is smaller. This is due to the weakness of the thermal-expansion effects, i.e. the decrease of the temperature difference.

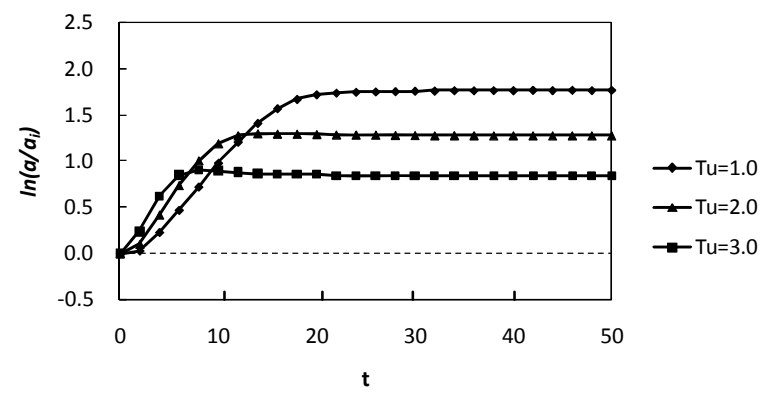

Fig. 5. Time histories of amplitude at $L e=1.0$ and $T_{\mathrm{u}}=1.0,2.0$ and 3.0
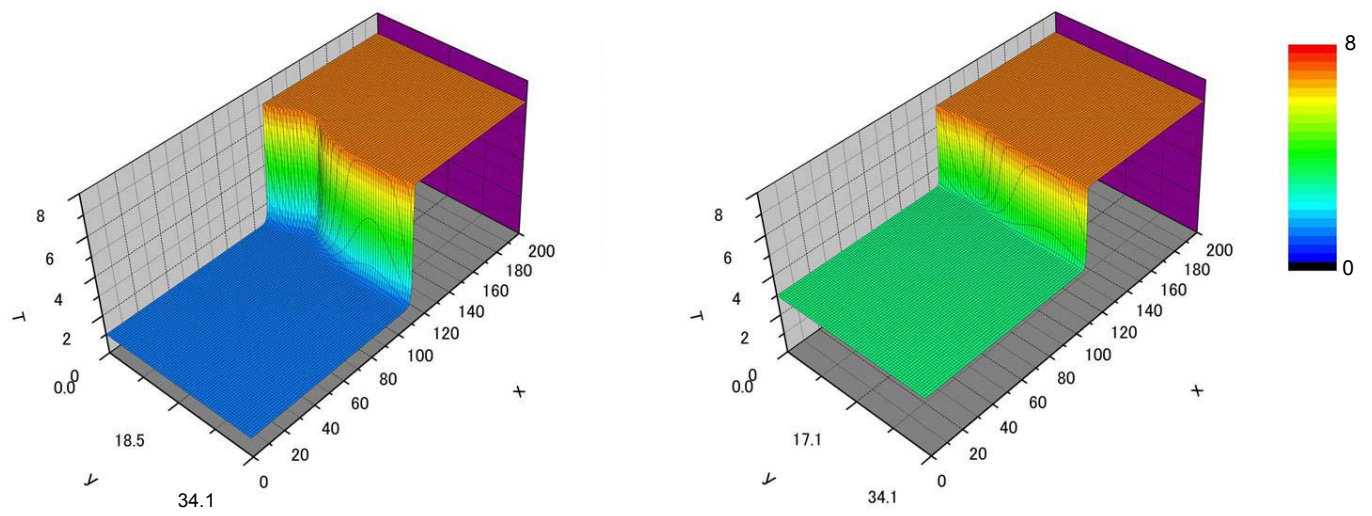

Fig. 6. Temperature distributions in $L e=1.0$ cellular flames at $T_{\mathrm{u}}=1.0 \& 3.0(t=40)$. 
The temperature distributions in $L e=1.0$ cellular flames at $T_{\mathrm{u}}=1.0 \& 3.0(t=40)$ are shown in Fig. 6, where the calculation domain in the $y$-direction is set equal to the critical wavelength $\lambda_{\mathrm{c}}=34.1$. The burned-gas temperature in downstream region is equal to the adiabatic flame temperature $\left(=T_{\mathrm{b}}=7.0\right)$. The unburned gas flows in from the left at the burning velocity of a planar flame, and the burned gas flows out to the right. The superimposed disturbance evolves owing to intrinsic instability, and then a cellular-shaped front forms. The cellular flame moves upstream, indicating that the burning velocity increases, which is due to the increase of flame-surface area. The increment of the burning velocity $\left(S_{\mathrm{cf}}-S_{\mathrm{u}}\right)$ is 0.24 and hardly changed, even though the unburned-gas temperature changes. In addition, the cell depth of the $T_{\mathrm{u}}=3.0$ flame, which is defined as the distance between the convex and concave flame fronts, is smaller than that of the $T_{\mathrm{u}}=1.0$ flame. This is because that the thermal-expansion effects become weaker owing to the decrease of the difference in temperature between the burned and unburned gases.
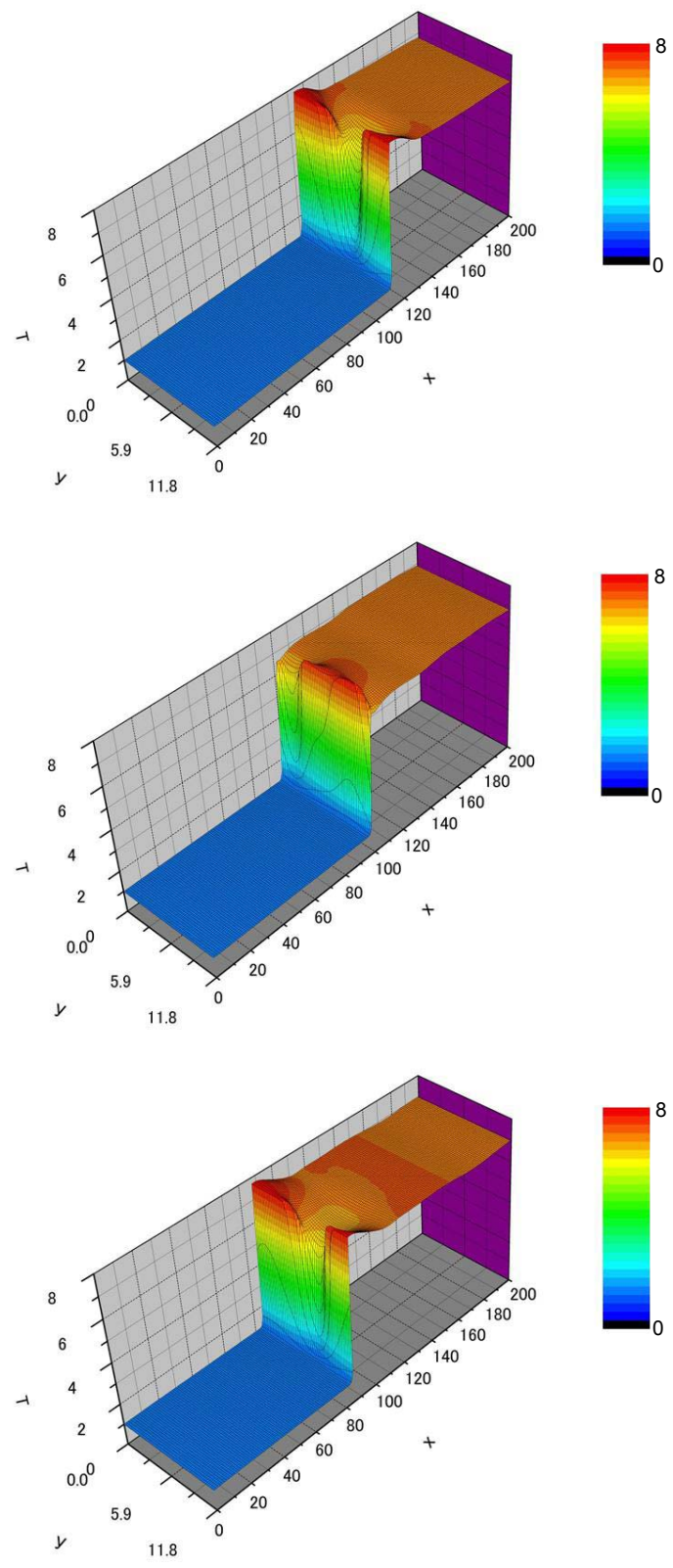

Fig. 7. Temperature distributions in $L e=0.5$ cellular flames at $T_{\mathrm{u}}=1.0(t=10,20$ and 30$)$. 

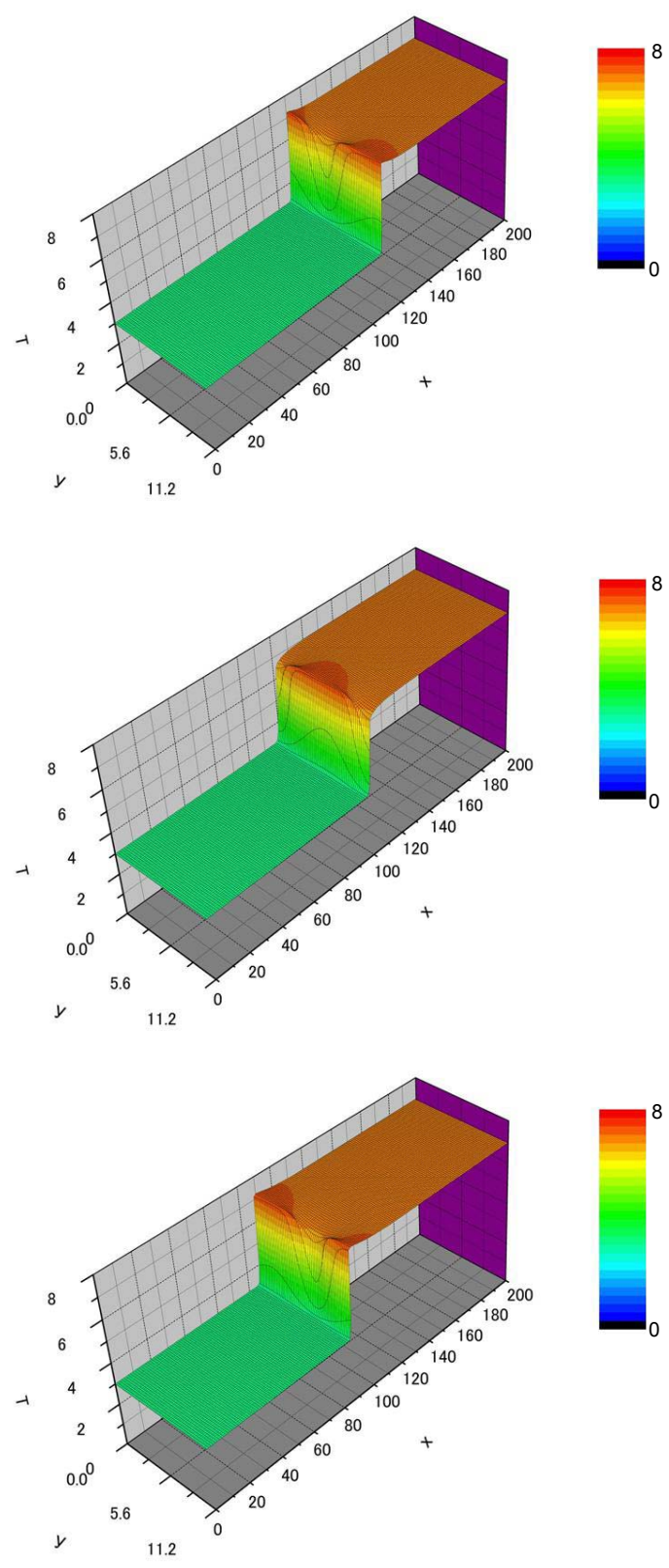

Fig. 8. Temperature distributions in $L e=0.5$ cellular flames at $T_{\mathrm{u}}=3.0(t=10,20$ and 30).

We change the Lewis number to investigate diffusive-thermal effects. The temperature distributions in $L e=0.5$ cellular flames at $T_{\mathrm{u}}=1.0$ and 3.0 are shown in Figs. 7 and 8 , respectively. Forming the cellular shape, the temperature at the convex flame front is higher than the adiabatic flame temperature. In addition, the cellular flame moves in the $y$-direction, i.e. moves laterally. This lateral movement is observed in $L e<1$ cellular flames. ${ }^{23}$ The laterally moving rate of cells at $T_{\mathrm{u}}=3.0$ is small compared with the $T_{\mathrm{u}}=1.0$ flame, even though the growth rate is great. This is due not only to the weakness of the thermal-expansion effects but also to the smallness of the Zeldovich number. The intensity of diffusive-thermal instability decreases as the Zeldovich number becomes smaller. The higher the unburned-gas temperature, the smaller the Zeldovich number. Thus, the instability intensity due to diffusive-thermal effects decreases as the unburned-gas temperature becomes higher. 
Figure 9 shows the temperature distributions in $L e=1.5$ cellular flames at $T_{\mathrm{u}}=1.0 \&$ $3.0(t=80)$. We find cellular-shaped fronts, where cells do not move laterally. This is because diffusive-thermal effects have a stabilizing influence at $L e>1$. Increased unburned-gas temperature causes mild behaviors of cellular flames, as the $L e=0.5$ and 1.0 cellular flames did.
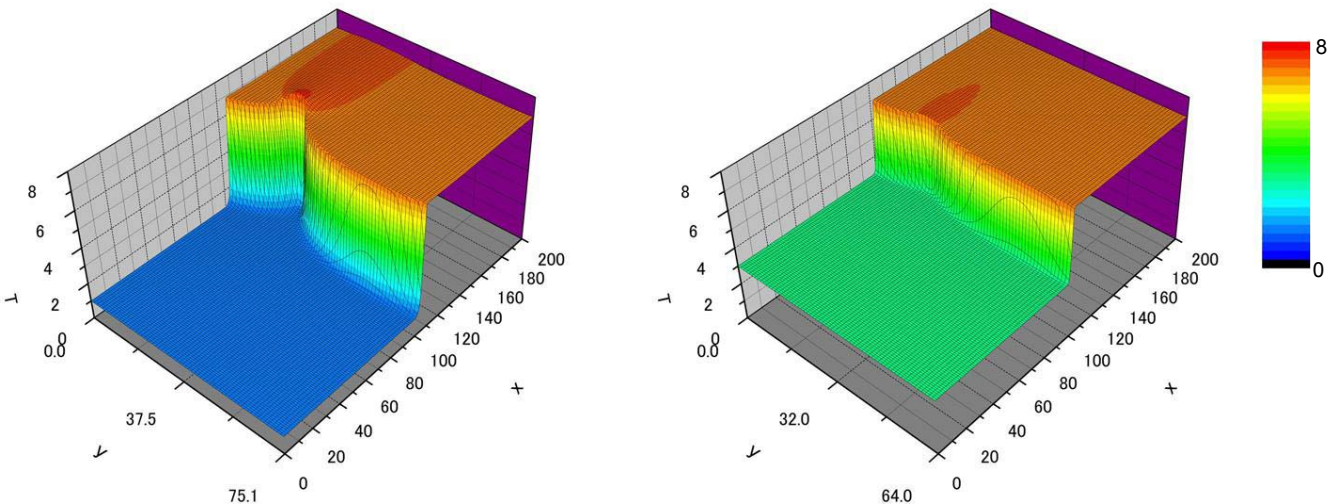

Fig. 9. Temperature distributions in $L e=1.5$ cellular flames at $T_{\mathrm{u}}=1.0 \& 3.0(t=80)$.

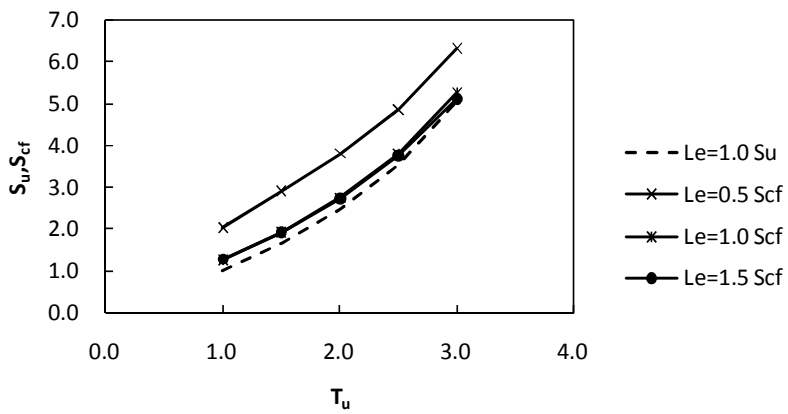

Fig. 10. Burning velocities of planar and cellular flames at $L e=0.5,1.0$ and 1.5, depending on the unburned-gas temperature.

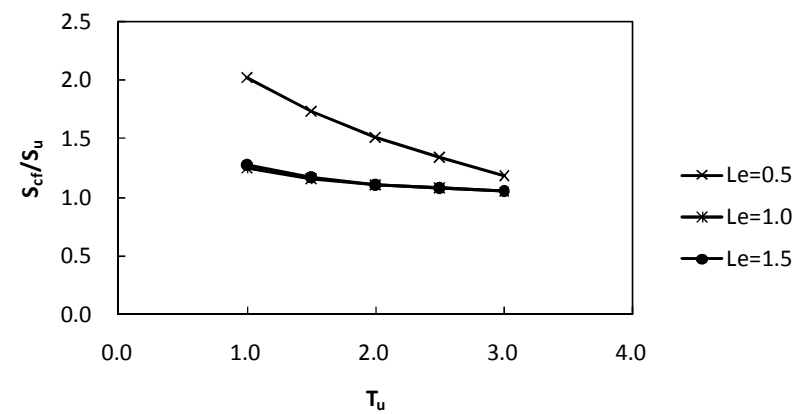

Fig. 11. Normalized burning velocities of cellular flames at $L e=0.5,1.0$ and 1.5, depending on the unburned-gas temperature. 
Next, we examine the burning velocity of a cellular flame. The value of the burning velocity is estimated as follows: The reaction rate of a cellular flame is integrated throughout the calculation domain, and the integrated value is divided by that of the $T_{\mathrm{u}}=$ 1.0 planar flame. The obtained value is equivalent to the burning velocity of a cellular flame.

Figure 10 shows the burning velocities of planar and cellular flames at $L e=0.5,1.0$ and 1.5, depending on the unburned-gas temperature. Both the burning velocities of planar and cellular flames increase drastically as the unburned-gas temperature becomes higher. Under conditions of the same Lewis numbers, the increment of the burning velocity ( $S_{\mathrm{cf}}$ $S_{\mathrm{u}}$ ) is nearly constant. Figure 11 shows the normalized burning velocities of cellular flames, i.e. $S_{\mathrm{cf}} / S_{\mathrm{u}}$. The normalized burning velocities decrease monotonously with an increase of the unburned-gas temperature. This is because that the burning velocities of planar and cellular flames increase drastically and that the increment is nearly constant.

\section{Conclusions}

In this paper, we have performed numerical calculations of two-dimensional unsteady reactive flows, based on the compressible Navier-Stokes equation, to investigate the effects of the unburned-gas temperature and Lewis number on the intrinsic instability of high-temperature premixed flames under the constant-enthalpy conditions, and have clarified the characteristics of cellular flames induced by intrinsic instability.

As the unburned-gas temperature becomes higher, the burning velocity of a planar flame increases drastically. This is because that the mass flux density increases and that the density of the unburned gas decreases under the constant-pressure conditions.

A sufficiently small disturbance is superimposed on a planar flame to investigate the intrinsic instability of high-temperature premixed flames. As the unburned-gas temperature becomes higher, the growth rate increases and the unstable range widens, because of the increase of the burning velocity. In addition, the obtained numerical results are consistent with the theoretical solutions in small wave-number range. As the Lewis number becomes smaller ( larger), the growth rate increases ( decreases ) and the unstable range widens ( narrows ), which is due to diffusive-thermal effects. The dispersion relation yields the critical wave number, and it increases as the unburned-gas temperature becomes higher. Thus, the critical wavelength shrinks, and then the cell size shrinks.

A finite disturbance with the critical wavelength is superimposed to clarify the characteristics of cellular flames. Evolving the disturbance, a cellular-shaped front forms. In all Lewis numbers, the behavior of cellular flames becomes milder as the unburned-gas temperature becomes higher, even though the growth rate increases. The normalized burning velocities of cellular flames decrease monotonously. This is because that the difference in temperature between burned and unburned gases decreases owing to the constant-enthalpy conditions.

\section{References}

(1) W. R. Dunbar and N. Lior, Sources of combustion irreversibility, Combustion Science and Technology, Vol. 103, p. 41, (1994).

(2) S. K. Som and A. Datta, Thermodynamic irreversibilities and exergy balance in combustion processes, Progress in Energy and Combustion Science, Vol. 34, p. 351, (2008). 
(3) Y. Yoshizawa, Thermodynamics of combustion with energy recirculation, Journal of the Combustion Society of Japan, Vol. 50, p. 111, (2008).

(4) K. Nishida, T. Takagi, and S. Kinoshita, Process analysis of exergy loss in combustion field, Transactions of the Japan Society of Mechanical Engineers, Series B, Vol. 68, p. 2643, (2002).

(5) F. J. Weinberg, Combustion temperature: the future?, Nature, Vol. 233, p. 239, (1971).

(6) T. Takeno, K. Sato, and K. Hase, A theoretical study on an excess enthalpy flame, Proceedings of the Combustion Institute, Vol. 18, p. 465, (1981).

(7) Y. Yoshizawa, K. Sasaki, and R. Echigo, Analytical study of the structure of radiation controlled flame, International Journal of Heat and Mass Transfer, Vol. 31, p. 311, (1988).

(8) K. Hanamura, R. Echigo, and S. A. Zhdanok, Superadiabatic combustion in a porous medium, International Journal of Heat and Mass Transfer, Vol. 36, p. 3201, (1993).

(9) M. Katsuki and T. Hasegawa, The science and technology of combustion in highly preheated air, Proceedings of the Combustion Institute, Vol. 27, p. 3135, (1998).

(10) T. Niioka, Fundamentals and applications of high temperature air combustion, Proceedings of the Fifth ASME/JSME Joint Thermal Engineering Conference, San Diego (1999).

(11) A. Cavaliere and M. de Joannon, Mild combustion, Progress in Energy and Combustion Science, Vol. 30, p. 329, (2004).

(12) Y. Miyagoshi, T. Tatefuku, M. Nishino, T. Yokoyama, and S. Kadowaki, Advantages of low air-ratio combustion in a stoker-type incinerator, Journal of Environment and Engineering, Vol. 2, p. 183, (2007).

(13) S. Kadowaki, Asymptotic analysis on high-temperature premixed flames: Instability of flame fronts under the constant-enthalpy conditions, Journal of Thermal Science and Technology, Vol. 5, p. 1, (2010).

(14) F. A. Williams, Combustion Theory, 2nd ed., Addison-Wesley, Reading, p.373, (1985).

(15) G. I. Sivashinsky, Diffusional-thermal theory of cellular flames, Combustion Science and Technology, Vol. 15, p. 137, (1977).

(16) G. Joulin and P. Clavin, Linear stability analysis of nonadiabatic flames: diffusional-thermal model, Combustion and Flame, Vol. 35, p. 139, (1979).

(17) P. Pelce and P. Clavin, Influence of hydrodynamics and diffusion upon the stability limits of laminar premixed flames, Journal of Fluid Mechanics, Vol. 124, p. 219, (1982).

(18) M. L. Franlel and G. I. Sivashinsky, The effect of viscosity on hydrodynamic stability of a plane flame front, Combustion Science and Technology, Vol. 29, p. 207, (1982).

(19) B. Denet and P. Haldenwang, Numerical study of thermal-diffusive instability of premixed flames, Combustion Science and Technology, Vol. 86, p. 199, (1992).

(20) A. Bayliss and B. J. Matkowsky, Nonlinear dynamivs of cellular flames, SIAM Journal of Applied Mathematics, Vol. 52, p. 396, (1992).

(21) B. Denet and P. Haldenwang, A numerical study of premixed flames Darrieus-Landau instability, Combustion Science and Technology, Vol. 104, p. 143, (1995).

(22) V. V. Bychkov, Propagation of curved stationary flames in tubes, Physical Review E, Vol. 54, p. 3713, (1996).

(23) S. Kadowaki, Numerical study on lateral movements of cellular flames, Physical Review E, Vol. 56, p. 2966, (1997).

(24) S. Kadowaki, The influence of hydrodynamic instability on the structure of cellular flames, Physics of Fluids, Vol. 11, p. 3426, (1999).

(25) S. Kadowaki and T. Hasegawa, Numerical simulation of dynamics of premixed flames: flame instability and vortex-flame interaction, Progress in Energy and Combustion Science, Vol. 31, p. 193, (2005). 
(26) G. J. Sharpe, Linear stability of planar premixed flames: reactive NavierStokes equations with finite activation energy and arbitrary Lewis number, Combustion Theory and Modelling, Vol. 7, p. 45, (2003).

(27) A. Kaewpradap and S. Kadowaki, The effects of the activation energy on the intrinsic instability of adiabatic and non-adiabatic premixed flames, Journal of Fluid Science and Technology, Vol. 3, p. 219, (2008). 